\title{
Performance Analysis of TCP and UDP over Mobile Ad hoc Network
}

\author{
Kashif Laeeq ${ }^{1}$, Noman Islam ${ }^{2}$, Jalal Bhayo ${ }^{3}$ \\ ${ }^{1,3}$ Department of Computer Science, National University of Computer \& Emerging Sciences, Karachi, Pakistan \\ ${ }^{2}$ Department of Computer Science, Iqra University, Karachi, Pakistan \\ ${ }^{1}$ kashiflaeeq@yahoo.com \\ ${ }^{2}$ noman. is lamegmail.com \\ ${ }^{3}$ jalalbhayo@gmail.com
}

\begin{abstract}
In the network computing domain, mobile ad hoc networks (MANETs) have gained prominence during the recent years. These networks have been used in almost all domains of today's life; especially in military and emergency operations. However, these application areas demand higher performance. It has been observed that better performance can't be achieved without improved network management and the detailed network performance awareness. In this direction, this research work investigates the behavior of mobile ad hoc network under two transport protocols i.e. Transmission Control Protocol (TCP) and User Datagram Protocol (UDP). The work analyzes various parameters such as bandwidth, throughput, jitter and out of order delivery etc. A test-bed has been established in university campus and the data transferred over the network is analyzed using iperf performance monitoring tool.
\end{abstract}

Keywords - ad hoc network; Iperf; performnce analysis; TCP; UDP.

\section{INTRODUCTION}

There are two types of wireless networks [1,2]. At one end, the wireless network can be established with the help of centralized entities called access points. The nodes communicate with each other via these access points. In the second category of wireless network i.e. wireless ad hoc network, the nodes discover and communicate with each other in peer to peer fashion without the help of any dedicated entity. In ad hoc network the nodes simultaneously work as a host as well as a router. The nodes are responsible themselves for different network operations. Sometimes ad hoc network can include fixed infrastructure as well. Such networks are called wireless mesh network (WMN). In WMN, there are stable nodes connected with each other in mesh fashions called as mesh routers. There are mesh clients that connect with mesh routers for communication purposes.

If the participating nodes of an ad-hoc network are all mobile and can move freely anywhere, they are called mobile ad hoc network (MANET). Such networks have been used for a wide range of applications such as rescue and relief operations, future combat systems, disaster management systems, virtual meetings, emergency operations and healthcare systems [1].

However, mobile ad-hoc networks are inherently prone to many challenges. Some of these challenges are medium access control, routing, addressing, security etc. [1-4]. Generally, these challenges arise due to scarcity of resources such as bandwidth, processing capability and storage. It becomes essential that various networking protocols operating in the mobile ad hoc network work in optimum fashion. There has been a lot of research going on to provide optimal algorithms for MANET.

This work analyzes the performance of transport protocols for MANET. The basic research question is to investigate the effect of mobility conditions on various performance attributes such as bandwidth, throughput, jitter and out of order delivery, for different transport protocols.

In conventional network, Transmission Control Protocol (TCP) and User Datagram Protocol (UDP) have been the most cited transport protocols. The former is a connection oriented protocol that is generally used for application demanding reliability. UDP is connection-less and unreliable protocol that are used for real-time application. TCP and UDP are standard network protocol on the internet and support large number of applications and communication needs, provide and network level consistency for multi-hop networks.

Despite of their wide use in conventional networks, these protocols don't perform optimally due to various unique properties of ad hoc network. This paper analyzes the two transport protocols i.e. TCP and UDP over ad hoc network. The iperf performance monitoring tool has been used for analyzing the performance of these protocols $[5,6]$. 
Before proceeding towards the discussion, the next section first presents the related work. Then details about the various experiments conducted to analyze the two protocols are provided. This is followed by the analysis and results of experiment. The paper concludes with discussion on future work.

\section{RELATED WORK}

There has been several studies reported in past about performance analysis of TCP and UDP under different ad hoc network routing protocols. In ad hoc network, the routing protocols can be either proactive such as Destination Sequenced Distance Vectoring Routing (DSDV) or reactive in nature such as Dynamic Source Routing (DSR), Ad hoc On-demand Distance Vector (AODV) routing and Temporally Ordered Routing (TORA). In the former category, routing paths are established periodically while in the latter category, a path is established only when needed for communication. In [7], performance of DSDV protocol under UDP and TCP traffic has been analyzed. Different performance parameters like packet loss, delay and pause time are observed for TCP and UDP traffic. Simulation results in [7] shown that TCP outperforms in high mobility environment while UDP gives better performance in less mobile scenario and denser network. According to [7], UDP traffic is better for high speed communication, while traffic loss can be decreased by setting high value of pause time.

In [8], the effect of route re-computation and high bit error rate on TCP performance in mobile ad hoc network is analyzed. Four routing protocols, DSDV, DSR, AODV and TORA are observed for experiments. Different variants of TCP protocols such as TCP Taho, TCP-Reno, TCP-New Reno, and TCP-Sack are used in simulations. TCP is evaluated for grid and chain topologies. In MANET, packet loss can be due to different reasons such as congestion and bit error. But TCP cannot identify exact reason and always invoke congestion control mechanism. In MANETs, dynamic topology rapidly changes route between sender and receiver. In [8], simulation results show that this algorithm reduces throughput, increases delays and severely degrades overall performance due to rapid link disconnections.

In [9], two on demand routing protocols namely AODV and DSR based on TCP traffic were analyzed. Routing performance is evaluated using TCP Sack and TCP New Reno. It is shown in [9] that TCP throughput is reduced when nodes move due to TCP's inability to differentiate between congestion and link failure. Congestion control and avoidance mechanism using Omni-antennas is proposed to optimize TCP for MANETS [9]. It is observed that with varying mobility conditions, DSR behaves more efficiently than other protocols. TCP Sack performance is slightly better than TCP New Reno.
In [10], UDP performance is analyzed using three routing schemes namely DSDV, AODV and DSR under varying number of nodes and mobility speed. Discrete event Network Simulator (NS2) from Berkley is used for analysis. It is observed that UDP throughput for DSDV is low because DSDV uses stale routing table entry in high mobility environment. DSR and AODV are reactive protocols, so throughput of them is better than DSDV.

\section{PERFORMANCE ANALYSIS OF TCP \& UDP ON AD-HOC NETWORK}

Improved network performance can't be attained without enriched management and the comprehensive network performance awareness. There are abundant network performance and monitoring assessment tools or techniques that help network specialist to look into network matters. The novelty of this experimental research lies in establishing a real world testbed and analyzing TCP and UDP performance in ad hoc network performance in various scenarios and context using iperf performance monitoring tool.

\section{A. Research method and experiment design}

The project performs an experiment comprising laptops that communicate with each other using TCP and UDP protocol. The experiment monitors the ad-hoc network via observing various parameters such as bandwidth, throughput, jitter and out of order delivery etc. The test-bed is established in a university campus. There are three laptops connected with each other via WiFi link. These laptops are running Microsoft Windows operating system. The laptops are mobile and move to various locations to transmit data-files of varying sizes. During each data transfer, performance properties are measured. These measured parameters are then analyzed further.

The Iperf server running on the particular system connects with the client. The detail of server side configurations are as follows:

$\$$ iperf -s

Server listening on TCP port 5001

TCP window size: $63.0 \mathrm{KByte}$ (default)

local 192.168.0.1 port 5001 connected with 192.168.0.2 port 49998

[ID] Interval Transfer Bandwidth

0.0-10.3 sec 6.25 MBytes 5.11 Mbits/sec

local 192.168.0.1 port 5001 connected with 192.168.0.2 port 50063 0.0-12.3 sec 7.12 MBytes 4.87 Mbits/sec

At the client side, different parameters can be used to analyze the TCP and UDP performance. To start the iperf client, simply provide the server IP address and port no on 
which the server is running, along with other parameters which are related to specific performance measurement of TCP and UDP. The detail of client side configurations is as follows:

\$ iperf-c 192.168.0.2

Client connecting to 192.168 .0 .2 , TCP port 5001

TCP window size: 63.0 KByte (default)

Local 192.168.0.1 port 55544 connected with 192.168.0.2 port 5001

[ID] Interval Transfer Bandwidth

0.0-10.1 sec 17.5 MBytes 14.6 Mbits/sec

The routing node receives data from other nodes and forwards it correspondingly. For this purpose, however, a route is added to forward the data to proper destination.

\section{B. Data Collection}

Different sets of information are collected for each of the two protocols. For TCP, the Maximum Segment Size (MSS), TCP window size, speed, packet loss, jitter and throughput are measured. For UDP, Maximum Transmission Unit (MTU), speed, loss, jitter and throughput, bandwidth delay product are measured. Figure 1-5 shows the results with TCP and UDP protocol.

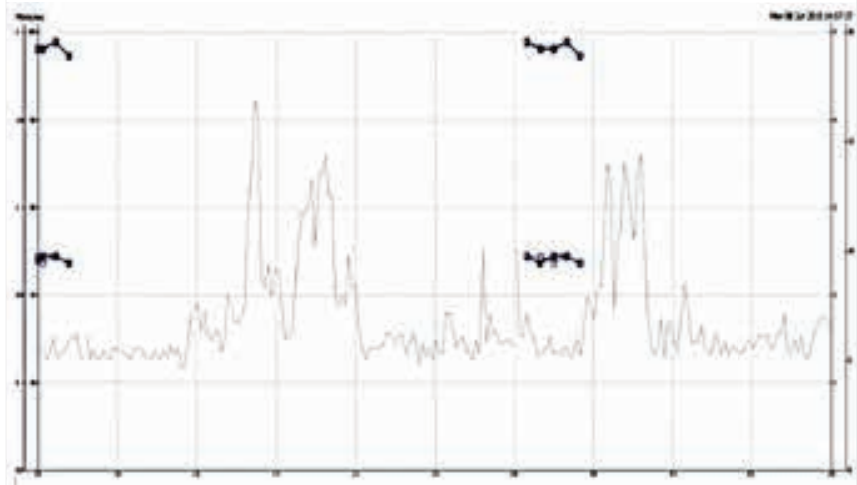

Fig. (1). Analysis ofUDP bandwidth parameter

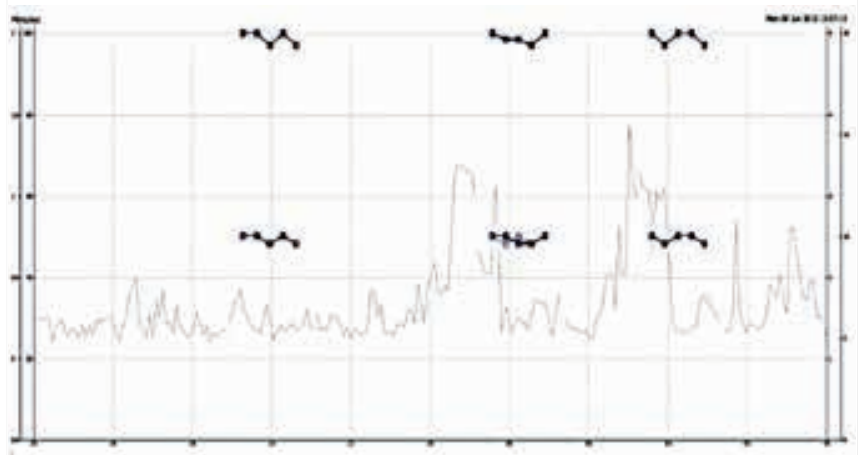

Fig. (2). Analysis of MSS parameter for TCP

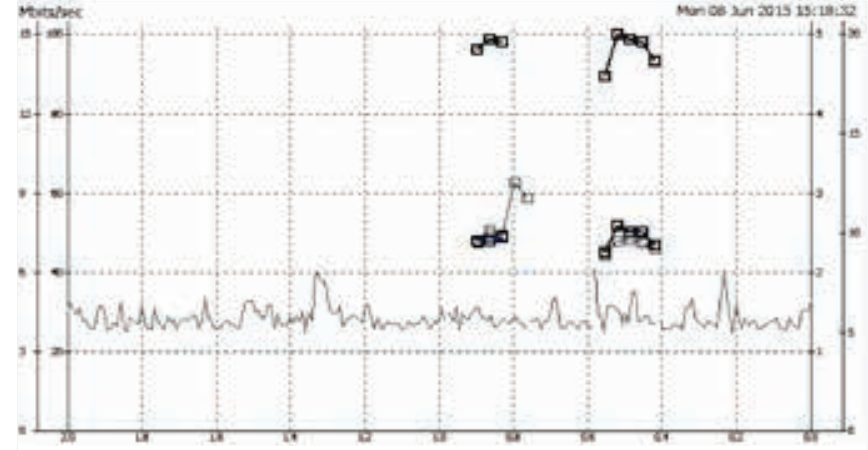

Fig. (3). Analysis of TCP windows size parameter

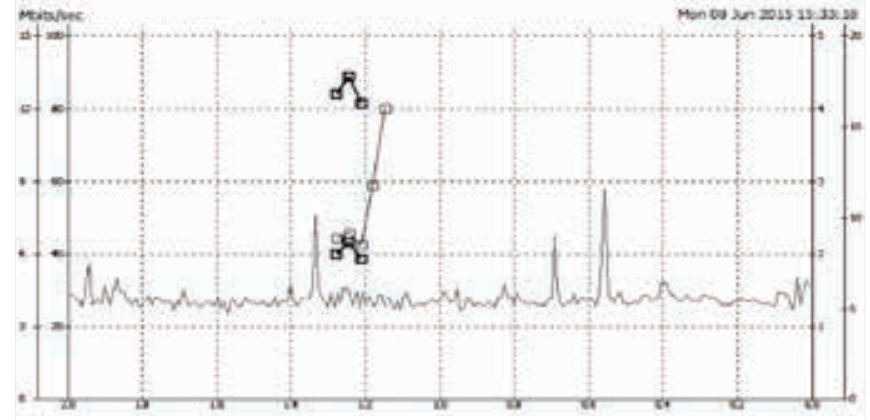

Fig. (4). Graph shows performance with TCP No delay after disabling the Nagle's Algorithm

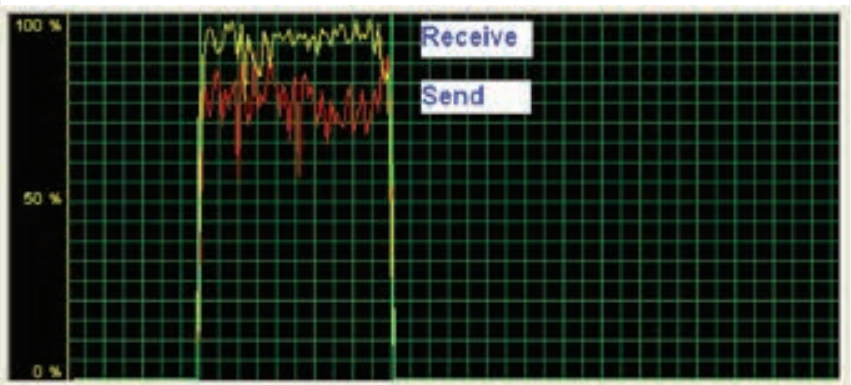

Fig. (5). Analysis of bidirectional data transfer simultaneously

\section{FUTURE WORK \& CONCLUSION}

In this work, the performance of TCP and UDP in ad hoc network performance monitoring has been analyzed using iperf performance monitoring tool. The outcome of this research can help network engineer for analysis, planning, design and implementation of large scale and complex network solutions considering different performance attributes of transport protocols. It also helps researchers in understanding of TCP and UDP behavior in ad hoc networking environments.

The novelty of this work lies in analysis and tuning the TCP and UDP performance through a popular performance analysis tool Iperf with various parameters. It is observed that higher node mobility or wider distance can affect the network performance. 
This research study highlights these issues and focused on the factors that degrade the ad-hoc network performance. The paper analyzed the bandwidth delay, throughput and TCP window size for monitoring the UDP and TCP performance with respect to congestion, packets loss and bandwidth. However, this work has been performed in a closed setting under limited nodes and low mobility. In order for a generalized conclusion to made, the same experiments have to be replicated for a large scale environment with very high mobility conditions. This exercise has been left as a future work.

Another dimension in which further research can be carried is to analyze different TCP variants such as TCP-Reno, TCP- Tahoe.

\section{REFERENCES}

[1] A. Jangra, N. Goel, P. and K. Bhatia, "Security aspects in mobile ad hoc networks (MANETs): A big picture" International Journal of Electronics Engineering, vol. 2, no. 1, pp: 189-196, 2010

[2] G. Sklyarenko, AODV Routing Protocol, the Seminar in Technische Informatik, Freie University, Berlin, Germany, 2006.

[3] B. Kannhavon. H. Nakayama, Y. Nemoto, N. Kato and A. Jamalipour; "A Survey of Routing Attacks in Mobile Ad Hoc Networks" IEEE Wireless Communications, vol. 1, no. 1, pp: 85-91, 2007.

[4] P. Yi, Z. Dai, Y. Zhong and S. Zhang," Resisting Flooding Attacks in Ad Hoc Networks", In Proceedings of the International Conference on Information Technology: Coding and Computing (ITCC'05), Las Vegas, April 4, 2005, pp. 657-662.
[5] A. Tirumala, L. Cottrell and T. Duningan, "Measuring end-to-end bandwidth with Iperf using Web100." In Proceedings of Passive and Active Monitoring Workshop (PAM 2003). 2003, pp. 1-8.

[6] M. Markus, C. Chambers, and S. J. Eggers. "Calpa: a tool for automating selective dynamic compilation." In Proceedings of the $33^{\text {rd }}$ annual ACM/IEEE International Symposium on Microarchitecture. Monterey, CA, USA, December, 2000. Pp: 291-302.

[7] Zahid, H. Farman, M. Ahmed, M. Najam-ul-Islam and H. Javed. "Performance Evaluation of TCP (Transmission Control Protocol) and UDP (User Datagram Protocol) over Destination Sequence Distance Vector (DSDV) for Random Waypoint Mobility Model." World Applied Sciences Journal, vol. 20, no. 7, pp: 910-916, 2012.

[8] F. Ahmed, S. K. Pradhan, N. Islam, and S. K. Debnath, "Performance Evaluation of TCP over Mobile Ad-hoc Networks," International Journal of Computer Science and Information Security, vol. 7, no. 1, pp: 140-146, 2010.

[9] K. Kathiravan, S. T. Selvi and A. Selvam, "TCP performance analysis for mobile adhoc network using on-demand routing protocols," Ubiquitous Computing and Communication Journal, pp: 370-376, 2007.

[10] B. C. Singh, T. K. Godder, M. S. Miah and H. M A. Awal, "Experimental Analysis of UDP performance in Mobile Ad Hoc Networks with different Routing protocol and varying payload" International Journal of Distributed and Parallel Systems, vol. 2, no. 6, pp: 107-113, 2011.

(C) Author(s) 2016. CC Attribution 4.0 License. (http://creativecommons.org/licenses/by-nc/4.0/)

This article is licensed under the terms of the Creative Commons Attribution Non-Commercial License which permits unrestricted, non-commercial use, distribution and reproduction in any medium, provided the work is properly cited. 